\title{
Ototoxicidad inducida por quimio-radioterapia basada en platinos: Una revisión
}

\author{
Ototoxicity induced by platinum compounds-based chemoradiotherapy: A review
}

Ignacio Cortés $\mathrm{F}^{1}$, María Celeste Casanova 0¹, Pía Michael L², Gerardo Oberreuter E².

\begin{abstract}
RESUMEN
Los análogos del platino, como el cisplatino, y la radioterapia son usados de forma individual y en conjunto para el tratamiento de diversas neoplasias en población adulta y pediátrica. Sin embargo, el uso de estos tratamientos puede generar ototoxicidad, especialmente cuando son usados de forma combinada para neoplasias que comprometen cabeza y cuello, manifestándose principalmente como una hipoacusia progresiva e irreversible que compromete la calidad de vida. Diversos mecanismos han sido propuestos para explicar el daño en las estructuras auditivas generado por estos tratamientos, incluyendo la producción de especies reactivas del oxígeno y la inflamación, desencadenando muerte celular. Si bien distintas estrategias otoprotectoras han sido probadas en humanos, es aún incierta su efectividad y seguridad en combinación con Ios tratamientos oncológicos. El objetivo de la siguiente revisión es proporcionar una visión general y actualizada de la ototoxicidad inducida por quimio-radioterapia basada en platinos, discutiendo sus bases, características clínicas, potenciales tratamientos y estrategias preventivas que se han desarrollado en los últimos años.
\end{abstract}

Palabras clave: Ototoxicidad, cisplatino, radioterapia, otoprotección.

\begin{abstract}
Platinum analogues, such as cisplatin, and radiotherapy are used separately or in combination to treat several neoplasms in pediatric and adult populations. Nonetheless, the use of these treatments may lead to ototoxicity, especially when these treatments are concomitantly used to treat head and neck cancers, which can manifest as progressive and irreversible hearing loss that decreases quality of life. Several mechanisms have been proposed in order to explain the damage to the auditory structures induced by these treatment modalities, including: reactive oxygen species production and inflammation, leading to cell death. Although several otoprotective strategies have been attempted in humans, their effectiveness and security are unclear. The objective of this review is to provide an updated and general overview on platinum-based chemoradio-
\end{abstract}

\footnotetext{
1 Escuela de Medicina, Universidad de Chile, Santiago, Chile.

2 Servicio de Otorrinolaringología, Hospital San Juan de Dios, Santiago, Chile.
}

Los autores declaran no tener conflictos de interés

Recibido el 4 de septiembre, 2018. Aceptado el 16 de diciembre, 2018. 
therapy induced ototoxicity, discussing its basis, clinical features, potential treatments and preventive strategies developed in recent years.

Key words: Ototoxicity, cisplatin, radiotherapy, otoprotection.

\section{INTRODUCCIÓN}

El uso de quimioterapia basada en compuestos derivados del platino y de radioterapia (RT), ya sea en conjunto o por separado, representa una aproximación común de tratamiento para distintos tipos de cáncer en niños ${ }^{1}$ y adultos ${ }^{2}$, incluyendo aquellos de base de cráneo, cabeza y cuello (CyC) con indicación de radiación ${ }^{3}$. Dentro de los análogos del platino, el cisplatino es uno de los compuestos más utilizados debido a su efectividad oncológica ${ }^{2}$. Por su parte, la RT es una importante modalidad de tratamiento y control de neoplasias ${ }^{4}$.

A grandes rasgos, el efecto antineoplásico de estos tratamientos está basado en la producción masiva de especies reactivas del oxígeno (EROs) y el daño irreversible al ADN, que puede resultar en muerte celular, principalmente por apoptosis, ${ }^{5,6}$. Por Io anterior, ejercen un efecto aditivo cuando son utilizadas en combinación $n^{3,7}$. El uso de ambos tratamientos está asociado a diversos efectos adversos, incluyendo ototoxicidad. En el caso de la RT, ésta se produce cuando estructuras auditivas se encuentran dentro del campo de acción en neoplasias de $\mathrm{CyC}^{7}$. El daño se puede manifestar clínicamente por hipoacusia sensorioneural (HSN), progresiva, irreversible, dosis-dependiente y que progresa desde las frecuencias agudas a las graves ${ }^{2,3,8-10}$. Además, la RT puede ocasionar daño a estructuras del oído medio, lo cual puede conllevar al desarrollo de otitis media con efusión (OME), agregando un componente conductivo a la pérdida auditiva ${ }^{11}$.

La prevalencia de ototoxicidad derivada de quimio-radioterapia (QRT) basada en platinos se ha estimado en hasta $90 \% 3,7,12$, dependiendo de las series, la edad y del uso concomitante de QRT basada en cisplatino. Se ha propuesto que esta considerable variabilidad en los resultados podría resultar, en parte, de las discrepancias en la definición de ototoxicidad y a la falta de escalas de evaluación estandarizadas de uso común, especialmente a nivel pediátrico ${ }^{13}$. En niños se ha establecido que la hipoacusia requiere intervenciones precoces y monitoreo clínico a largo plazo, por la importancia de la audición en el desarrollo psicosocial y cognitivo $0^{12,14,15}$. En adultos, la hipoacusia puede conllevar a una disminución de la calidad de vida ${ }^{2,7}$, cuya rehabilitación involucra un alto costo económico para los sistemas de salud ${ }^{16}$. Para prevenir la aparición de ototoxicidad se han intentado distintas estrategias incluyendo: la reducción de dosis durante los tratamientos combinados de QRT; dosis fraccionadas de RT, la RT de intensidad modulada, evitar incluir las estructuras auditivas en el campo de radiación ${ }^{3}$; y estrategias farmacológicas para reducir el daño generado en células normales por estos tratamientos ${ }^{17-19}$, las que han presentado efectividad variable y la incertidumbre de que potencialmente puedan reducir la efectividad oncológica de estos tratamientos. En consecuencia, no existe ningún tratamiento farmacológico preventivo o terapéutico aprobado oficialmente para tratar este efecto adverso a la fecha, lo que ha sido motivo de creciente investigación en los últimos años.

El objetivo de la presente revisión es proporcionar una visión general y actualizada de la ototoxicidad inducida por QRT basada en platinos, discutiendo sus bases, características clínicas, potenciales tratamientos y estrategias preventivas que se han desarrollado en los últimos años.

\section{Quimio-radioterapia basada en platinos}

Los análogos del platino consisten en una molécula con un átomo de platino en su centro rodeada de diferentes sustituyentes que otorgan distintos perfiles de acción y toxicidad, siendo el oxaliplatino y el carboplatino menos ototóxico que el cisplatino ${ }^{20}$. El efecto antineoplásico está dado por la unión irreversible del platino al ADN formando aductos ${ }^{5}$, junto con una significativa producción de EROs gracias a la disfunción mitocondrial gatillada por el platino ${ }^{21}$, lo que genera daño en las biomoléculas, desencadenando muerte celular principalmente por apoptosis ${ }^{5}$.

La RT ejerce sus efectos antineoplásicos por medio del daño directo al genoma, resultando en 
rupturas simples o dobles de las cadenas del ADN 6 . Sin embargo, la mayoría del daño al ADN sería de forma indirecta a través de la producción de EROs. La radiación interactúa con las moléculas de agua de las células, ionizándolas, formando una serie de productos como radical hidroxilo, peróxido de hidrógeno que pueden generar daño oxidativo en distintos objetivos críticos dentro de las células, como las membranas, proteínas y ADN, desencadenando muerte celular ${ }^{22}$. Otros efectos de la RT incluyen el desarrollo de inflamación y estrés oxidativo crónico tisular23.

\section{Mecanismos de ototoxicidad generados por la quimio-radioterapia basada en platinos}

Si bien los detalles específicos de cómo la QRT genera ototoxicidad no han sido completamente dilucidados, diversos estudios preclínicos han demostrado que los análogos del platino son capaces de generar cantidades elevadas de EROs en las células ciliadas externas, principalmente radical anión superóxido, siendo uno de los mecanismos principales de ototoxicidad ${ }^{5}$. Además del daño generado al ADN nuclear, se ha visto que el cisplatino generaría daño al ADN mitocondrial, incrementando la producción de EROs de una forma independiente al daño directo al ADN nuclear provocado por la generación de aductos ${ }^{21}$.

Se ha reportado que la isoforma NOX3 de la enzima NADPH oxidasa, que cataliza la generación de superóxido, tiene una alta expresión en el órgano de Corti y en las células del ganglio espiral. Esta enzima incrementaría su producción de superóxido luego de la exposición a platinos, lo cual ha mostrado tener un rol importante en la fisiopatología de la ototoxicidad ${ }^{24,25}$. Otras alteraciones que han sido reportadas incluyen: alteraciones del calcio ${ }^{26}$; depleción de los sistemas antioxidantes ${ }^{5}$; y la formación de peroxinitrito, una especie reactiva del nitrógeno de alta potencia que puede generar inactivación de proteínas ${ }^{27}$.

Los mecanismos específicos de daño auditivo generados por la RT son aún menos caracterizados que los por cisplatino. In vitro, la radiación puede generar un incremento de EROs en células derivadas del órgano de Corti de una forma dosisdependiente ${ }^{28}$. Este efecto sería más pronunciado en las células ciliadas externas de las porciones basales de cóclea que en las apicales, debido a niveles menores del antioxidante glutatión en las células basales ${ }^{29}$.

Además, otros efectos de la RT incluyen la generación de un estado proinflamatorio y prooxidante crónico que ocurriría meses 0 incluso años después de la exposición, generando muerte celular y fibrosis ${ }^{22,23}$, lo cual aún no ha sido caracterizado en el oído interno. No obstante, la RT puede generar cambios inflamatorios en el oído medio que conllevan al desarrollo de $\mathrm{OME}^{11}$.

Ambas modalidades de tratamiento han demostrado generar daño auditivo por medio de la inducción de apoptosis. No obstante, la QRT ha mostrado incrementar la expresión de genes relacionados a la apoptosis comparado a cada tratamiento por sí solo en células cocleares ${ }^{30}$, 10 que sería concordante con la mayor frecuencia de ototoxicidad observada en pacientes que reciben esquemas combinados ${ }^{3}$.

\section{Características clínicas de la ototoxicidad}

La ototoxicidad generada por los compuestos derivados del platino clínicamente se caracteriza por presentar hipoacusia sensorioneural, bilateral, frecuentemente progresiva e irreversible, dosisdependiente, asociándose en grado variable a tinnitus y síntomas vestibulares 2,3,-10. Típicamente compromete primero las frecuencias agudas y progresa hacia las graves de forma dosisdependiente ${ }^{2}$. Esta pérdida auditiva puede ser temprana, produciéndose desde las primeras horas hasta días luego de la administración de cisplatino². No obstante, es posible observar pérdida auditiva años después de la terapia, lo cual podría estar asociado a cambios generados por la retención de los compuestos platinados en el cuerpo a largo plazo $^{31}$.

Los factores de riesgo descritos para desarrollar ototoxicidad por análogos del platino incluyen: el tipo de platino, siendo el cisplatino más ototóxico que el carboplatino y el oxaliplatino ${ }^{20}$; la dosis total utilizada ${ }^{32}$; las edades extremas; el uso concomitante de RT que incluye la $\mathrm{CyC}^{3}$; trauma auditivo; hipoacusia previa; falla renal; y asociación con otros ototóxicos como aminoglicósidos y furosemida ${ }^{2,33}$. Además, se han asociado distintos factores genéticos, como polimorfismos de genes 
involucrados en el transporte y procesamiento de los platinos en el oído ${ }^{34,35}$.

La RT puede también por sí sola generar daño en diversas estructuras de la vía auditiva cuando éstas están comprendidas en las áreas de radiación ${ }^{11}$. Así, en el oído externo es posible observar lesiones agudas como eritema, descamación e infrecuentemente lesiones ulceradas, y crónicas como atrofia cutánea, ulceración, estenosis del canal y disminución de la producción de cerumen por destrucción glandular. En el oído medio se puede generar edema y disfunción tubárica desencadenando OME, daño en la cadena osicular, agregando un componente conductivo a la hipoacusia. La manifestación más importante de la ototoxicidad atribuible a RT es la HSN, la cual se origina en el daño de distintas estructuras del oído interno ${ }^{11}$, incluso afectando el nervio auditivo y vías centrales 0 estructuras vestibulares ${ }^{36}$. En el oído afectado por RT, la pérdida auditiva tiene características similares a la gatillada por cisplatino ${ }^{3}$.

Si bien alrededor del mundo existen distintos reportes sobre las características de la ototoxicidad, estudios realizados en población chilena son aún escasos. En un estudio prospectivo piloto de 6 pacientes con neoplasias malignas de CyC tratados con QRT con cisplatino en 3 ciclos $\left(100 \mathrm{mg} / \mathrm{m}^{2}\right)$, donde la dosis de RT no fue reportada, se observó una disminución en las emisiones otoacústicas (EOA) evocadas transitorias y las de productos de distorsión, con predominio en las frecuencias agudas y de instalación precoz (24 horas), ocurriendo antes de la aparición de síntomas ${ }^{37}$.

Recientemente, Waissbluth y collis en un estudio de cohorte prospectiva, evaluaron los efectos de la QRT con platinos en la audición a largo plazo en 12 niños con distintos tipos de cáncer tratados en el Complejo Asistencial Dr. Sótero del Río. De este grupo de niños, 7 recibieron cisplatino, 2 carboplatino, 3 ambas drogas y 5 recibieron RT concomitante. Se reportó que $6(50 \%)$ de los pacientes desarrolló hipoacusia temprana luego de la quimioterapia y 7 (58,3\%) a largo plazo durante el seguimiento (casi 12 años), siendo la mayoría clínicamente significativa, definida como aquella $\geq 40 \mathrm{~dB}$ a frecuencias iguales 0 superiores a $4.000 \mathrm{~Hz}$ ( $\geq 2$ a según la clasificación de Chang) ${ }^{38}$. Cuatro pacientes empeoraron su audición durante el seguimiento y 2 pacientes recibieron audífonos bilaterales. Ocho $(66,7 \%)$, cinco $(41,7 \%)$ y dos $(16,7 \%)$ pacientes desarrollaron tinnitus, vértigo recurrente y desequilibrio, respectivamente y 3 $(25 \%)$ experimentaron alteraciones en la prueba de video impulso cefálico. Además, se reportaron distintas alteraciones en cuestionarios relacionados a reconocimiento de discurso, ubicación espacial y calidad de audición. Si bien este estudio presentó algunas limitaciones como el tamaño muestral reducido, se constituye como un esfuerzo importante de caracterización de la ototoxicidad en Chile, recomendando al menos 10 años de seguimiento clínico de estos pacientes.

Uno de los principales desafíos actuales ha sido lograr establecer un sistema de evaluación y clasificación de la ototoxicidad que sea de uso común, existiendo actualmente un gran número de escalas disponibles ${ }^{7,13}$. Desde el punto de vista operacional, estas escalas difieren en distintos aspectos tales como: la inclusión de frecuencias agudas $>4.000 \mathrm{~Hz}$; el valor en $\mathrm{dB}$ para considerar una hipoacusia significativa; complejidad en su aplicación; la sensibilidad y especificidad para detectar la necesidad de rehabilitación auditiva ${ }^{13,39}$. Existen diversas dificultades para el desarrollo de escalas comunes, tales como: alta variabilidad en los perfiles clínicos de los pacientes incluyendo la edad, la presencia o no de lenguaje previo al tratamiento (lo cual influye en la modalidad de evaluación audiométrica), el deterioro funcional originado por la enfermedad oncológica antes o durante el tratamiento, las dificultades técnicas con los equipos de audiología (movilización de pacientes para realizarse las evaluaciones), el desconocimiento de las características de la ototoxicidad y la falta de comunicación entre el equipo oncológico y el otorrinolaringólogo. En suma, esto ha influido en la variabilidad reportada en la incidencia de ototoxicidad y aún más importante, en la posibilidad de identificar a los afectados y entregar rehabilitación auditiva oportuna ${ }^{7,13,39}$.

\section{Seguimiento y manejo}

El objetivo del seguimiento y manejo es identificar tempranamente alteraciones auditivas e intervenir oportunamente, reduciendo el impacto en la calidad de vida. Si bien existen guías clínicas que entregan orientaciones generales sobre el seguimiento de 
pacientes con ototoxicidad ${ }^{40,41}$, en los últimos años se ha avanzado en el desarrollo y actualización de dichas prácticas, especialmente aquellas que conciernen al seguimiento a largo plazo, la inclusión de otras técnicas para la detección de ototoxicidad, y también la rehabilitación precoz, especialmente en pacientes pediátricos ${ }^{42}$. A continuación se resumen algunas recomendaciones basadas en revisiones recientes ${ }^{2,7,39,43,44}$. Antes del inicio del tratamiento oncológico, se debe realizar una evaluación basal que incluya: anamnesis detallada que contenga la presencia de factores de riesgo para desarrollar ototoxicidad; otoscopía; impedanciometría; audiometría convencional extendida de las frecuencias agudas (6.000-8.000 $\mathrm{Hz}$ ). En niños menores a 5 años, debido a las dificultades de realizar audiometría convencional, se debería evaluar los productos de distorsión de EOA, debido a que son más fáciles de obtener y han demostrado correlación con daño auditivo precoz debido a platinos ${ }^{45,46}$. En algunos casos se podría incluir la realización de potenciales evocados auditivos cuando estén disponibles y sean factibles de realizar de acuerdo al estado clínico del paciente.

Idealmente, la evaluación clínica y audiométrica debiera realizarse después de cada ciclo de cisplatino (especialmente cuando existen factores de riesgo de ototoxicidad asociados) 0 cada 2-4 ciclos de carboplatino. Para aquellos que reciben sólo RT, se recomienda una evaluación al completar la terapia. Una vez finalizado el tratamiento oncológico, se recomienda una evaluación seriada a los 3, 6, 9, 12 meses, luego anualmente, especialmente en pacientes pediátricos. Se sugiere que estos pacientes deberían seguirse por un plazo de al menos 5-10 años, especialmente si recibieron RT concomitante ${ }^{39,42}$. En el caso de los adultos el seguimiento podría ser menos frecuente y de menor duración, detectando oportunamente el momento de iniciar la rehabilitación ${ }^{40,44}$.

En cuanto a las intervenciones a realizar, el rol del otorrinolaringólogo en esta patología incluye: el seguimiento clínico del paciente en relación a la aparición de dificultades auditivas, trastornos del equilibrio y tinnitus; comunicación del grado de hipoacusia al equipo oncológico tratante para evaluar la posibilidad de modificación de terapia, lo cual no siempre es posible debido a las características de la enfermedad de base; y ofrecer alternativas de dispositivos de amplificación ya sea audífonos y/0 implantes cocleares. Además, se debería evaluar la posibilidad de utilización de dispositivos que permitan estimulación eléctrica y acústica en aquellos pacientes que presenten compromiso de un amplio rango de frecuencias.

\section{Estrategias de prevención de ototoxicidad}

Dado que la QRT con platinos genera muerte celular oxidativa, distintas moléculas han sido probadas con el fin de reducir la ototoxicidad, especialmente antioxidantes. No obstante, la mayoría de la evidencia proviene de estudios preclínicos basados en cisplatino, siendo aún limitada la evidencia en humanos. Más aún, considerando que los efectos antineoplásicos de estas modalidades de tratamiento surgen de mecanismos similares a los de ototoxicidad, aún persisten ciertas dudas sobre la potencial interferencia en la efectividad oncológica al usar un tratamiento otoprotector sistémico concomitante. Esta potencial interacción se ha mantenido como una de los principales obstáculos en el desarrollo de estrategias otoprotectoras sistémicas y ha propiciado el estudio de estrategias locales de prevención. Se discutirán principalmente aquellas estrategias que han sido probadas en humanos.

\section{Estrategias sistémicas}

En un estudio con 36 adultos con tumores malignos cerebrales tratados por hasta 1 año con carboplatino intraarterial (dosis total $400 \mathrm{mg} / \mathrm{m}^{2}$ ) y apertura osmótica de la barrera hémato-cerebral mensual, donde 17 recibieron tiosulfato de sodio (TSS) sistémico 4 horas después de cada ciclo de carboplatino, y 19 no lo recibieron. La incidencia de ototoxicidad fue menor (29\%) en aquellos pacientes que recibieron TSS comparado con los que no recibieron el antioxidante (84\%) durante el seguimiento ${ }^{47}$. Este mismo compuesto fue evaluado recientemente en población pediátrica, en un ensayo clínico abierto fase III, aleatorizado, controlado y multicéntrico ${ }^{18}$. Los pacientes fueron asignados aleatoriamente a recibir TSS $16 \mathrm{~g} / \mathrm{m}^{2}$ endovenoso 6 horas después de cada dosis de cisplatino $(n=49)$ o a no recibir TSS después de la quimioterapia $(n=55)$. Después del ajuste 
por variables de estratificación tales como edad ( $<5$ años $0 \geq 5$ años), duración de la infusión de cisplatino ( $<2$ h $0 \geq 2$ h), RT craneal, el riesgo de pérdida auditiva fue menor en el grupo que recibió TSS (Odds ratio [OR] 0,31; 95\% intervalo de confianza [IC] 0,13-0,73; $p=0,0036$ ). No obstante, aquellos pacientes con enfermedad más avanzada que recibieron TSS presentaron menor sobrevida libre de eventos a los 3 años (42\%; 95\% IC 0,21$0,61)$ comparado al grupo control $(61 \%, 95 \%$ IC $0,39-0,77$ ) (p por log-rank test $<0,05$ ) y una menor sobrevida promedio $(45 \%, 95 \%$ IC $0,23-0,65)$ comparado a los controles $(84 \%, 95 \%$ IC $0,62-$ 0,94).

Recientemente, en un ensayo clínico prospectivo, aleatorizado y multicéntrico, se probó el uso de TSS en pacientes pediátricos con hepatoblastoma de riesgo estándar tratados con cirugía y cisplatino $^{19}$. Los participantes fueron aleatoriamente asignados a recibir cisplatino y cirugía $(n=52), 0$ cisplatino, cirugía y TSS $(n=57) 6$ horas después de terminada la infusión de cisplatino, por 4 ciclos preoperativos y 2 ciclos posoperativos. Los autores reportaron que aquellos pacientes que recibieron TSS presentaron menor incidencia de pérdida auditiva de grado $\geq 1$ ( $\geq 40 \mathrm{~dB}$ a frecuencias iguales o superiores a $8.000 \mathrm{~Hz}$ ) de acuerdo a la escala de Brock $^{48}$ comparado al grupo solo con cisplatino (riesgo relativo: 0,52; 95\% IC 0,33-0,81; $p=0,002$ ). Además, después de 52 meses de seguimiento, no se observaron diferencias significativas en la sobrevida promedio ni en la sobrevida libre de eventos a 3 años entre los grupos. Si bien ambos estudios fueron similares en cuanto a metodología, las poblaciones de pacientes fueron distintas ya que en el primer estudio se incluyeron niños con diversos tipos de cáncer y en el segundo solo con hepatoblastoma, por lo que la comparación en cuanto a sobrevida debe ser cautelosa.

Se probó el uso de n-acetilcisteína (NAC) oral en un ensayo clínico aleatorizado controlado con placebo realizado en el Hospital San Juan de Dios con 45 pacientes que recibieron QRT con cisplatino como tratamiento para cánceres de CyC. Setenta y tres por ciento de los pacientes desarrollaron algún grado de HSN en la audiometría de alta frecuencia $(6.000-16.000 \mathrm{~Hz})$ al finalizar el tratamiento ${ }^{49}$. Aquellos que recibieron NAC oral $(n=23)$ durante el tratamiento experimentaron una mejoría parcial, expresado en un menor grado de HSN de altas frecuencias durante el seguimiento comparado al grupo placebo $(n=22)$. Cabe destacar que el seguimiento fue menor a un año, no se reportaron efectos adversos asociados al fármaco y este estudio corresponde a una de las primeras experiencias de este tipo en población chilena.

Otro compuesto tiólico que se ha probado es la amifostina. Un metaanálisis reciente mostró que no existe evidencia suficiente para recomendar el uso de amifostina como estrategia preventiva en niños con osteosarcoma 0 hepatoblastoma'. No obstante, la amifostina sí ha mostrado tener cierto rol en la reducción de la severidad de xerostomía, mucositis o disfagia por RT, aparentemente sin interferir en la efectividad de los tratamientos oncológicos de dichos pacientes ${ }^{50}$. No obstante, si bien esta droga ha sido probada en modelos animales de ototoxicidad por $\mathrm{RT}^{51}$ dichos efectos en humanos aún no son explorados.

Se ha reportado que la vitamina $E$ podría reducir el grado de ototoxicidad asociado a platinos ${ }^{52}$. En un ensayo aleatorizado controlado con placebo, los pacientes suplementados con vitamina $E$ ( $n$ 13) no presentaron diferencias significativas, medidas pos y prequimioterapia, en los umbrales auditivos a $2.000,4.000$ y $8.000 \mathrm{~Hz}$ comparado al grupo placebo $(n=10)$ al primer mes de seguimiento, el cual exhibió un empeoramiento auditivo medido a los 2.000 y $8.000 \mathrm{~Hz}$. No existieron diferencias significativas en los potenciales evocados de tronco entre ambos grupos ${ }^{53}$. Aun cuando se mostró cierto efecto potencialmente otoprotector, este estudio presentó diversas limitaciones metodológicas tales como: una alta tasa de pérdida de pacientes las cuales no fueron mayormente detalladas, la inexistencia de análisis de sobrevida y un seguimiento de sólo 1 mes. En pacientes con cánceres de CyC, también se ha explorado el uso de vitamina $\mathrm{E}$ para prevenir efectos asociados a la RT. En un estudio aleatorizado doble ciego controlado con placebo ${ }^{54}$, el uso combinado de vitamina $E$ y $C(n=25)$ previo a la $R T$ se asoció a una menor incidencia de xerostomía a los 6 meses comparado al grupo placebo $(n=20)$. En otro estudio similar, el uso de vitamina E previo a cada sesión de RT se asoció a una menor incidencia de mucositis sintomática durante las 7 semanas de tratamiento ${ }^{55}$. Si bien en estos 2 estudios no se 
reportaron diferencias en la sobrevida, en otros 2 ensayos aleatorizados prospectivos diseñados para reducir efectos adversos asociados a la RT de CyC mediante el uso de vitamina $\mathrm{E}$ y betacaroteno, sí se reportó peor sobrevida en aquellos pacientes que recibieron vitaminas ${ }^{56,57}$.

\section{Estrategias locales}

Considerando la potencial interferencia entre terapias sistémicas con la efectividad oncológica de la QRT, es que se han propuesto estrategias de administración local de sustancias en el oído con el fin de reducir la hipoacusia posterior a los tratamientos oncológicos. Esto se basaría en la potencial difusión de drogas desde el oído medio al interno a través de la ventana redonda, lo que ha mostrado un posible rol en el tratamiento de algunas enfermedades del oído interno como la enfermedad de Ménière y la HSN súbita ${ }^{58,59}$. En un estudio piloto en 20 pacientes con cánceres de CyC con indicación de cisplatino, el uso de infusión transtimpánica de NAC al $10 \%$ en un oído antes de la infusión de cisplatino en cada ciclo mostró reducir el cambio en umbrales auditivos al final de Ios ciclos ${ }^{60}$. En otro estudio similar con NAC al $2 \%$ administrado 30-60 minutos antes de cada ciclo de cisplatino, no hubo diferencias significativas a los 2 meses de evaluación ${ }^{61}$.

Se probó el uso de dexametasona transtimpánica en un oído (el otro oído fue usado como control), en un estudio prospectivo aleatorizado controlado en 15 pacientes con distintos tipos de cáncer tratados con cisplatino, previo a cada sesión de quimioterapia. Se observó que aquellos oídos que recibieron dexametasona experimentaron un menor grado de pérdida auditiva a 6.000 $\mathrm{Hz}$ y una reducción de los productos de distorsión de EOA en el rango de 4.000 a $8.000 \mathrm{~Hz}$. En relación al uso de estrategias protectoras locales en pacientes tratados con RT, la evidencia clínica es limitada, existiendo reportes de uso de dexametasona en modelos in vitro e in vivo con resultados positivos ${ }^{62,63}$.

Aunque las estrategias locales de administración de sustancias se constituyen como una alternativa interesante y potencialmente efectiva para disminuir la ototoxicidad asociada a QRT por platinos, su uso aún se encuentra en fases experi- mentales. Esto debido a limitaciones tales como: comportamiento farmacocinético desconocido de las sustancias en el oído en relación a la dosis necesaria, tiempos de acción, pérdida a través de la tuba auditiva, difusión intracoclear, diferencias anatómicas de la ventana redonda; posibilidad de inflamación y OME causado por RT que impida la absorción del fármaco; y tolerancia del procedimiento ${ }^{64,65}$. Sin embargo, en los últimos años diversas estrategias de administración local se han desarrollado, incluyendo diversos tipos de materiales como polímeros que prolongan la liberación de fármacos en el tiempo, lo cual podría facilitar el uso de este tipo de estrategias de prevención de ototoxicidad ${ }^{58,66}$.

\section{CONCLUSIONES}

El uso de quimioterapia basada en platinos y de RT es frecuente debido a su eficacia antineoplásica. La ototoxicidad se presenta como un efecto adverso importante y frecuente de estos tratamientos. Los mecanismos detrás de su desarrollo son complejos y no bien caracterizados molecularmente, siendo la generación masiva de EROs uno de los más aceptados. Clínicamente este daño se expresa principalmente como HSN irreversible, dosisdependiente que progresa desde las frecuencias agudas a las graves. La ototoxicidad es una patología que representa varios desafíos actualmente, entre ellos los problemas de clasificación y pesquisa; el requerir intervenciones multidisciplinarias, sobre todo en niños; y la necesidad de un seguimiento clínico frecuente y a largo plazo.

Si bien se ha logrado reconocer como un evento adverso frecuente tanto en población adulta como pediátrica, el potencial impacto en la calidad de vida de esta patología ha sido subestimado. Esto toma especial énfasis en niños y aquellos pacientes que reciben tratamientos con intención curativa y que, por lo tanto, poseen un mayor riesgo de desarrollar deterioro auditivo secundario al tratamiento a lo largo de su vida. Actualmente no existe ningún tratamiento farmacológico preventivo o terapéutico para tratar este efecto adverso, siendo un tópico de interés creciente en los últimos años dado el potencial impacto que tiene en la calidad de vida de los pacientes 
y en los sistemas de salud con relación a los costos asociados a la rehabilitación auditiva. Una de las principales dificultades para el desarrollo de estrategias farmacológicas para prevenir esta patología ha sido la posibilidad de interferir con la efectividad oncológica de los tratamientos. En este punto la evidencia sobre el uso de TSS es controversial, no existiendo estudios que corroboren esta información en otros escenarios clínicos tales como el uso de RT concomitante o cánceres en etapas más avanzadas. Si bien la administración local de medicamentos en el oído parece ser una alternativa razonable para proveer otoprotección sin interferir en los tratamientos oncológicos, la evidencia en relación a su uso para prevenir

\section{BIBLIOGRAFÍA}

1. van As JW, van den Berg $H$, van Dalen EC. Medical interventions for the prevention of platinuminduced hearing loss in children with cancer. Cochrane Database Syst Rev 2016; 2016: CD009219.

2. Paken J, Govender CD, Pillay M, Sewram V. Cisplatin-Associated Ototoxicity: A Review for the Health Professional. J Toxicol 2016; 2016: 1-13.

3. Theunissen ear, Bosma SCJ, Zuur CL, et al. Sensorineural hearing loss in patients with head and neck cancer after chemoradiotherapy and radiotherapy: A systematic review of the literature. Eisele DW, ed. Head Neck 2015; 37: 281-92.

4. Yap ML, Zubizarreta E, Bray F, Ferlay J, Barton M. Global Access to Radiotherapy Services: Have We Made Progress During the Past Decade? J Glob Oncol 2016; 2: 207-15.

5. Sheth S, Mukherjea D, Rybak LP, Ramkumar V. Mechanisms of Cisplatin-Induced Ototoxicity and Otoprotection. Front Cell Neurosci 2017; 11.

6. Powell S, McMillan TJ. DNA damage and repair following treatment with ionizing radiation. Radiother Oncol 1990; 19: 95-108.

7. LandieR W. Ototoxicity and cancer therapy. Cancer 2016; 122: 1647-58.

8. Frisina RD, Wheeler he, Fossa SD, et al. Comprehensive audiometric analysis of hearing impairment and tinnitus after cisplatin-based ototoxicidad asociada a QRT por platinos sigue siendo limitada. No obstante, la existencia de experiencia clínica en relación a la administración transtimpánica de fármacos en algunas patologías del oído y el desarrollo de distintos tipos de biomateriales y tecnologías ha propiciado el estudio de la administración local de medicamentos como una alternativa potencialmente factible para reducir la ototoxicidad asociada a QRT con platinos.

Por último, la caracterización de esta patología en población chilena es aún limitada, por lo que su conocimiento se vuelve necesario, y considerando lo expuesto previamente, de crucial importancia para el desarrollo de protocolos nacionales de manejo y seguimiento de estos pacientes.

chemotherapy in survivors of adult-onset cancer. J Clin Oncol 2016; 34: 2712-20.

9. Clemens E, de Vries AC, am Zehnhoff-Dinnesen $A$, ET AL. Hearing loss after platinum treatment is irreversible in noncranial irradiated childhood cancer survivors. Pediatr Hematol Oncol 2017; 34: $120-9$

10. Bhandare N, Jackson A, Elsbruch A, et al. Radiation therapy and hearing loss. Int J Radiat Oncol Biol Phys 2010; 76: S50-7.

11. Jereczek-Fossa BA, Zarowski A, Milani F, Orecchia R. Radiotherapy-induced ear toxicity. Cancer Treat Rev 2003; 29: 417-30.

12. van As JW, van den Berg H, van Dalen EC. Platinuminduced hearing loss after treatment for childhood cancer. In: van As JW, ed. Cochrane Database of Systematic Reviews. Chichester, UK: John Wiley \& Sons, Ltd; 2016.

13. Waissbluth S, Peleva E, Daniel SJ. Platinuminduced ototoxicity: a review of prevailing ototoxicity criteria. Eur Arch Oto-RhinoLaryngology 2017; 274: 1187-96.

14. Brock PR, KNIGHt KR, Freyer DR, et al. PlatinumInduced Ototoxicity in Children: A Consensus Review on Mechanisms, Predisposition, and Protection, Including a New International Society of Pediatric Oncology Boston Ototoxicity Scale. J Clin Oncol 2012; 30: 2408-17.

15. Waissbluth S, Chuang A, Del Valle Á, Cordova $M$. Long term platinum-induced ototoxicity in pediatric patients. Int J Pediatr Otorhinolaryngol 2018; 107: 75-9. 
16. Mohr PE, Feldman JJ, Dunbar JL, et al. The societal costs of severe to profound hearing loss in the United States. Int J Technol Assess Health Care 2000; 16: 1120-35.

17. Chen J, ZHAO Y, Zhou X, et AL. Methylprednisolone use during radiotherapy extenuates hearing loss in patients with nasopharyngeal carcinoma. Laryngoscope 2016; 126: 100-3.

18. Freyer DR, Chen L, Krailo MD, et al. Effects of sodium thiosulfate versus observation on development of cisplatin-induced hearing loss in children with cancer (ACCL0431): a multicentre, randomised, controlled, open-label, phase 3 trial. Lancet Oncol 2017; 18: 63-74.

19. Brock PR, Maibach R, Childs M, et al. Sodium Thiosulfate for Protection from Cisplatin-Induced Hearing Loss. N Engl J Med 2018; 378: 2376-85.

20. Rabik CA, Dolan ME. Molecular mechanisms of resistance and toxicity associated with platinating agents. Cancer Treat Rev 2007; 33: 9-23.

21. Marullo R, Werner E, Degtyareva N, et al. Cisplatin induces a mitochondrial-ROS response that contributes to cytotoxicity depending on mitochondrial redox status and bioenergetic functions. PLoS One 2013; 8: e81162.

22. Mujica-Mota MA, Lehnert S, Devic $S$, Gasbarrino K, Daniel SJ. Mechanisms of radiation-induced sensorineural hearing loss and radioprotection. Hear Res 2014; 312: 60-8.

23. Zнао W, RoвbINS MEC. Inflammation and chronic oxidative stress in radiation-induced late normal tissue injury: therapeutic implications. Curr Med Chem. 2009; 16: 130-43.

24. Rybak LP, Mukherjea D, Jajoo S, Kaur T, Ramkumar V. siRNA-mediated knock-down of NOX3: therapy for hearing loss? Cell Mol Life Sci. 2012; 69: 2429-34.

25. Bámfi B, Malgrange B, Knisz J, Steger K, DuboisDauphin M, Krause K-H. NOX3, a superoxidegenerating NADPH oxidase of the inner ear. $J$ Biol Chem 2004; 279: 46065-72.

26. Mukherjea D, Jajoo S, Whitworth C, et al. Short Interfering RNA against Transient Receptor Potential Vanilloid 1 Attenuates CisplatinInduced Hearing Loss in the Rat. $J$ Neurosci 2008; 28: 13056-65.

27. Jamesdaniel S, Manohar S, Hinduja S. Is S-nitrosylation of cochlear proteins a critical factor in cisplatin-induced ototoxicity? Antioxidants Redox Signal 2012; 17: 929-33.

28. Low W-KK, TAn MGK, Sun L, Chua AWC, Goh L-KK, WANg D-YY. Dose-dependant radiation-induced apoptosis in a cochlear cell-line. Apoptosis 2006; 11: 2127-36.

29. Sha SH, Taylor R, Forge A, Schacht J. Differential vulnerability of basal and apical hair cells is based on intrinsic susceptibility to free radicals. Hear Res 2001; 155: 1-8.

30. Low W-K, Kong SWW, Tan MGK. Ototoxicity from combined Cisplatin and radiation treatment: an in vitro study. Int J Otolaryngol 2010; 2010: 523976.

31. Breglio AM, Rusheen AE, Shide ED, et al. Cisplatin is retained in the cochlea indefinitely following chemotherapy. Nat Commun 2017; 8: 1654.

32. LI Y, Womer RB, SILber JH. Predicting cisplatin ototoxicity in children: the influence of age and the cumulative dose. Eur J Cancer 2004; 40: 2445-51.

33. Bokemeyer C, Berger CC, Hartmann JT, et al. Analysis of risk factors for cisplatin-induced ototoxicity in patients with testicular cancer. $\mathrm{Br}$ J Cancer 1998; 77: 1355-62.

34. Mukherjea D, Rybak LP. Pharmacogenomics of cisplatin-induced ototoxicity. Pharmacogenomics 2011; 12(7): 1039-50.

35. Waissbluth S, Daniel SJ. Cisplatin-induced ototoxicity: Transporters playing a role in cisplatin toxicity. Hear Res 2013; 299: 37-45.

36. GIBB AG, LoH KS. The role of radiation in delayed hearing loss in nasopharyngeal carcinoma. $J$ Laryngol Otol 2000; 114: 139-44.

37. Irany PM, Codjambassis D, Pinto J. Emisiones Otoacústicas en la Detección Precoz de Ototoxicidad Inducida por Cisplatino. Rev Otorrinolaringol Cir Cabeza Cuello 2000; 60: 7-13.

38. Chang KW, Chinosornvatana N. Practical grading system for evaluating cisplatin ototoxicity in children. J Clin Oncol 2010; 28: 1788-95.

39. Bass JK, Bhagat SP. Challenges in Ototoxicity Monitoring in the Pediatric Oncology Population. J Am Acad Audiol 2014; 25: 760-74.

40. ASHA. Audiologic Management of Individuals Receiving Cochleotoxic Drug Therapy. Med Pediatr Oncol March 1994: 1-17.

41. Durrant JD, Campbell KM, Fausti SA, et al. American Academy of Audiology Position 
Statement and Clinical Practice Guidelines Ototoxicity Monitoring American Academy of Audiology Position Statement. 2009: 1-25.

42. Children's Oncology Group. Long-Term Follow-Up Guidelines for Survivors of Childhood, Adolescent, and Young Adult Cancers. Version 4.0. Children's Oncology Group. Monrovia (CA). 2013.

43. Brooks B, KNIGHT K. Ototoxicity monitoring in children treated with platinum chemotherapy. Int J Audiol 2018; 57: S34-40.

44. Konrad-Martin D, Poling GL, Garinis AC, et al. Applying U.S. national guidelines for ototoxicity monitoring in adult patients: perspectives on patient populations, service gaps, barriers and solutions. Int J Audiol 2018; 57: S3-S18.

45. Knight KR, Kraemer DF, Winter C, Neuwelt EA. Early Changes in Auditory Function As a Result of Platinum Chemotherapy: Use of Extended HighFrequency Audiometry and Evoked Distortion Product Otoacoustic Emissions. J Clin Oncol 2007; 25: 1190-5.

46. Dhooge I, Dhooge C, Geukens S, De Clerck B, De Vel E, Vinck BM. Distortion product otoacoustic emissions: An objective technique for the screening of hearing loss in children treated with platin derivatives. Int J Audio/ 2006; 45: 337-43.

47. Doolittle ND, Muldoon LL, Brummett RE, et al. Delayed sodium thiosulfate as an otoprotectant against carboplatin-induced hearing loss in patients with malignant brain tumors. Clin Cancer Res 2001; 7: 493-500.

48. Brock PR, Bellman SC, Yeomans EC, Pinkerton CR, Pritchard J. Cisplatin ototoxicity in children: A practical grading system. Med Pediatr Oncol 1991; 19: 295-300.

49. Muñoz D, Cardemil F, Readi A, Contreras JM. Protective role of n-acetylcisteine from cisplatininduced ototoxicity in patients with head and neck cancer. A randomized, placebocontrolled clinical trial. In: $6^{\text {th }}$ World Congress of the International Federation of Head and Neck Oncologic Societies. Buenos Aires, Argentina; 2018.

50. GU J, ZHu S, LI X, WU H, LI Y, HuA F. Effect of amifostine in head and neck cancer patients treated with radiotherapy: A systematic review and meta-analysis based on randomized controlled trials. Gires 0, ed. PLOS One 2014; 9: e95968.
51. Lessa RM, Oliveira jaA De, Rossato M, Ghilardi Netto T. Analysis of the cytoprotective effect of amifostine on the irradiated inner ear of guinea pigs: an experimental study. Braz $J$ Otorhinolaryngol 2009; 75: 694-700.

52. Kalkanis JG, Whitworth C, Rybak LP. Vitamin E Reduces Cisplatin Ototoxicity. Laryngoscope 2004; 114: 538-42.

53. Villani V, Zucchella C, Cristalli G, et al. Vitamin E neuroprotection against cisplatin ototoxicity: Preliminary results from a randomized, placebo-controlled trial. Head Neck 2016; 38: E2118-E2121.

54. Chung MK, Kıм DH, Aнn YC, Chо JY, Kıм EH, Son YI. Randomized trial of Vitamin C/E complex for prevention of radiation-induced xerostomia in patients with head and neck cancer. Otolaryngol Head Neck Surg 2016; 155: 423-30.

55. Ferreira PR, Fleck JF, Diehl A, et al. Protective effect of alpha-tocopherol in head and neck cancer radiation-induced mucositis: A double-blind randomized trial. Head Neck 2004; 26: 313-21.

56. Bairati I, Meyer F, Jobin E, et al. Antioxidant vitamins supplementation and mortality: A randomized trial in head and neck cancer patients. Int J Cancer 2006; 119: 2221-4.

57. Bairati I, Meyer F, Gélinas M, et al. Randomized Trial of Antioxidant Vitamins to Prevent Acute Adverse Effects of Radiation Therapy in Head and Neck Cancer Patients. J Clin Oncol 2005; 23: 5805-13.

58. Musazzı UM, Franzé S, Cilurzo F. Innovative pharmaceutical approaches for the management of inner ear disorders. Drug Deliv Transl Res 2018; 8: 436-49.

59. Nguyen K, Kempfle JS, Jung DH, McKenna CE. Recent advances in therapeutics and drug delivery for the treatment of inner ear diseases: a patent review (2011-2015). Expert Opin Ther Pat 2017; 27: 191-202.

60. Riga MG, Chelis L, Kakolyris S, et al. Transtympanic Injections of N-acetylcysteine for the Prevention of Cisplatin-induced Ototoxicity. Am J Clin Oncol 2013; 36: 1-6.

61. Yoo J, Hamilton SJ, Angel D, et al. Cisplatin otoprotection using transtympanic L-Nacetylcysteine: A pilot randomized study in head and neck cancer patients. Laryngoscope 2014; 124: 1-8. 
62. Dinh CT, Chen S, Dinh J, et al. Effects of Intratympanic Dexamethasone on High-Dose Radiation Ototoxicity In Vivo. Otol Neuroto/2017; 38: 180-6.

63. Dinh C, Chen S, Padgett K, et al. Dexamethasone Protects Against Radiation-induced Loss of Auditory Hair Cells In Vitro. Otol Neurotol 2015; 36: $1741-7$.

64. Salt AN, PlontKe SK. Principles of Local Drug
Delivery to the Inner Ear. Audiol Neurotol 2009; 14: $350-60$.

65. Swan EEL, Mescher MJ, Sewell WF, Tao SL, Borenstein JT. Inner ear drug delivery for auditory applications. Adv Drug Deliv Rev 2008; 60: 1583-99.

66. El Kechai N, Agnely F, Mamelle E, Nguyen Y, Ferrary E, Воснот A. Recent advances in local drug delivery to the inner ear. Int J Pharm 2015; 494: 83-101. 